\title{
La cultura del trabajo en precario: hacia una sociedad desarticulada
}

\author{
Yolanda Bravo Vergel
}

\section{Resumen}

Desde la perspectiva del asociacionismo y la práctica democrática se analiza la participación de los jóvenes en la cultura del trabajo y el efecto potencialmente desarticulador de éste en las sociedades avanzadas.

Palabras clave: asociacionismo, teoría democrática, cultura del trabajo.

Abstract. Precarious' labor culture: Towards a disjointed's society

From the perspective of asociationism and democratic practice, the article analyses youth participation in the labour culture. The potencial disarticulating effect of labour in advanced societies is also examined.

Key words: asociotionism, democratic theory, labour culture.

\section{Sumario}

1. Participación política, asociacionismo y teoría democrática

2. Jóvenes y asociacionismo
3. El "problema del trabajo»: ¿desarticulador social?

Algunas conclusiones

Bibliografia

Las asociaciones parecen organizaciones simples [...] pero realmente son un microcosmos donde se encierran todas las complejidades de nuestras sociedades. Precisamente por eso, nos parecen interesantes, como elementos intermediarios entre lo micro y lo macro de la sociedad, como intercambio de una serie de actividades que no son ni del Estado ni del mercado, como proceso cotidiano entre el voluntariado, los profesionales y los usuarios, etc. (Villasante, 1993: 9).

Las asociaciones voluntarias han sido consideradas por teóricos de la sociedad de masas, como Kornhauser, parte necesaria de la infraestructura democrática: 
son lo bastante cercanas al individuo para permitirle alguna clase de participación activa, y pueden ser lo bastante cercanas al Estado como para posibilitar algún tipo de influencia política real. En otras palabras, según esta teoría la existencia de asociaciones voluntarias aumenta el potencial democrático de una sociedad: los miembros organizados están más inclinados a ser ciudadanos activos y más comprometidos con los valores democráticos ${ }^{1}$.

Almond y Verba (1963) han demostrado que al margen de la clase de integración -asociación pasiva o asociación en una organización no política- la afiliación tendrá un efecto positivo sobre la competencia política, siendo de esperar aumente el potencial para el compromiso y la actividad políticas. El afiliado está, por consiguiente, más cerca del tipo ideal del ciudadano democrático. Si el pluralismo es uno de los fundamentos más importantes de la democracia, el asociacionismo es uno de los pilares del pluralismo.

$\mathrm{El}$ análisis que realizamos pretende averiguar si los mensajes y los valores económicos - competitividad, eficiencia, individualismo-y la estructura social fragmentada que se está perfilando afectan o afectarán negativamente en un futuro al ya de por sí "asociacionismo absentista" de los jóvenes, y en general de toda la población.

\section{Participación política, asociacionismo y teoría democrática}

La democracia entraña, por definición, participación popular. La participación es la clave de bóveda en la construcción de una sociedad verdaderamente democrática. La pregunta es ¿qué grado de participación es necesario?

How extensive must social and economic pluralism be? All of the matters referred to are matters of degree, they are nor susceprible to precise measurement (Pennock, 1979: 221).

Tampoco es posible contestar con una única respuesta a la pregunta de jes deseable que aumente la participación? Karl Marx fue el portavoz de la maximización de la participación, como única vía para dotar al alienado de identidad en el seno de su comunidad. Descle otro extremo, se advierte qué niveles elevados de participación pueden tener un efecto desestabilizador sobre el sistema, aunque el sentimiento de competencia sí desempeña un importante papel en la estabilidad democrática. Esta es la postura de Almond y Verba (1963), que no consideran a los altos niveles de participación como expresión ideal de su modelo de cultura cívica, apostando por un ciudadano "templado» ${ }^{2}$. No obstante, las razones a favor son varias e importantes. En primer

1. KORNHAUSER, William, The Politics of Mass Society, Glencoe, I11, 1959.

2. Almond y Verba (1963) definen la cultura cívica como una "cultura leal de participación en la que son congruentes la cultura y las estructuras políticas". Una cultura cívica descansa sobre un conjunto de actitudes - actitudes genéricas frente a los demás, sentimiento de confranza social-y filiaciones - de grupo primario- no políticas. 
lugar, la más simple y obvia es mejorar la producción de outputs del sistema: las políticas públicas. En segundo lugar, la participación activa tiende a aumentar el sentimiento de legitimidad hacia el sistema, con consecuencias favorables tanto para la democracia en sí como para los propios ciudadanos - evitando la frustración y la alienación del individuo.

Actualmente, algunos teóricos de la democracia participativa anteponen la importancia de los efectos directos de la participación sobre el propio ciudadano por encima de los beneficios para el sistema. Esta línea de argumentación no es nueva: John Stuart Mill, por no hablar de Rousseau o Aristóteles, ya defendieron la corresponsabilización del individuo con la tareas de gobierno como vía más efectiva para contribuir a su desarrollo moral e intelectual. Pero, ‘a través de qué mecanismos puede un ciudadano participar en las actuales sociedades de masas? La respuesta de J.S. Mill es la organización de la población en pequeñas unidades: las asociaciones de ciudadanos.

J.S. Mill alabó los beneficios educativos y morales del asociacionismo. Tocqueville, su valiosa contribución a la libertad en las sociedades democráticas.

Town meetings are to liberty what primary schools are to science: they bring it within the people's reach and teach how to use and how to enjoy it (A. Tocqueville, Democray in America, 2: 61).

Alexis de Tocqueville, uno de los padres de la sociología política, fue el primer gran representante de la idea de que la democracia implica un equilibrio entre las fuerzas de conflicto y consenso. La preocupación de Tocqueville por un sistema político pluralista resultó de su interpretación de las tendencias de las sociedades modemas de masas: la concentración del poder en el Estado leviatán podría generar una situación de progresivo uniformismo y atomización del individuo, en nombre de la democracia. El debate que abre Tocqueville - que le convertirá en un antecesor de los críticos de la "Sociedad de masas» ${ }^{3}$ advierte de cómo el desarrollo de las tendencias igualadoras podría no conducir a una sociedad de individuos más libres, integrados y creativos sino, por el contrario, a una sociedad más gris, mediocre y apática. El individuo atomizado, sin pertenencia a una unidad social políticamente significativa, carecería de los canales, la información y el interés suficientes para participar en la política. La sociedad, desarticulada, caería en el riesgo del despotismo de la mayoría y la individualidad, la apatía de unos ciudadanos sin identidad colectiva, minaría el propio significado de la democracia.

Su estudio sobre la democracia en los Estados Unidos le sugirió dos instituciones que podrían combatir estos riesgos: el autogobiemo local y las asociaciones voluntarias. Éstas, como fuente de control y restricciones sobre el

3. En la teoría de la sociedad de masas el individuo queda configurado como relacionado directamente, en cuanto a individuo, con el Estado. No posee otras relaciones sociales que le apoyen en esta relación y naturalmente se siente ineficaz y lleno de ansiedad. 
gobierno, como "escuelas de libertad", articulan la sociedad, movilizando el poder en pequeñas unidades de modo que los individuos sean conscientes de su propia competencia política y puedan recoger los frutos de la participación que Mills describe: el desarrollo de sus capacidades intelectuales y morales. Tocqueville recalca cómo la participación en tales colectivos locales y asociaciones es determinante para la estabilidad del sistema democrático: pueden crear y mantener el consenso necesario en una sociedad democrática.

[...] no son los elementos institucionales, sino sobre todo elementos de tipo social, insertados en las costumbres, en lo que después se llamará "cultura política" los que pueden hacer posible el dificil objetivo de compaginar la democracia y la libertad (Botella y otros, 1994: 381).

Pero las asociaciones no sólo son pieza necesaria para crear y mantener el consenso en democracia. También son necesarias para introducir el cambio y la renovación, la acción colectiva transformadora frente al sistema o el aparato institucional, porque son las "constructoras" de nuevos valores desde sus propias prácticas cotidianas, al tiempo que soportes de los movimientos sociales (Villasante, 1993). Ya sean movimientos de onda corta (movilizaciones), de onda media (populares) o de onda larga (históricos), para pasar de unos a otros son necesarias las asociaciones porque sólo ellas pueden darles continuidad, estando sus identidades en continua renovación, bebiendo del pulso popular: ahí está la clave de la riqueza y la creatividad asociativa.

\section{Jóvenes y asociacionismo}

En toda sociedad existen gran variedad de organizaciones, que podríamos sintetizar como estatales, con ánimo de lucro y sociales. Nosotros nos centraremos en las organizaciones sociales (sin ánimo de lucro) propiamente llamadas asociaciones (independientes y constituidas por voluntarios). La definición que Tomás Alberich propone en su tipología es la siguiente: «Agrupación independiente de personas constituida para realizar una actividad colectiva estable -asociaciones de vecinos, consumidores, juveniles, APAS, deportivas, de mujeres, sindicatos, ONG, etc. $\longrightarrow$ (Alberich, 1993: 54).

Tratando de no caer en el mismo error que los estudios de opinión destinados a configurar «retratos robot» de los jóvenes - como si fueran un colectivo homogéneo-, partiremos siempre de la base de que la juventud no es una esencia en sí misma (Feixa: 1993), sino que es vivida por cada joven en función del contexto en que se halle. Centraremos nuestro análisis en la juventud catalana, entendida en un sentido amplio entre los 15 y los 29 años de edad, pero haremos referencias constantes a la realidad de la juventud española en su conjunto. Compararemos ambos colectivos con el conjunto de la población catalana y/o española cuando lo consideremos relevante.

El interés manifestado por el asociacionismo entre los jóvenes catalanes es, en general, alto. Así se desprende de la Enquesta a la Joventut de Catalunya 1990. 
Tabla 1. Pertenencia de los jóvenes a una asociación

\begin{tabular}{lclc}
\hline & Sí & No & NS/NC \\
\hline Total & $27,8 \%$ & $71,8 \%$ & $0,4 \%$ \\
\hline Sexe & & & \\
\hline Home & 32,3 & 67,6 & 0,1 \\
Dona & 23,0 & 76,3 & 0,6 \\
\hline
\end{tabular}

Edat

\begin{tabular}{llll}
\hline $15-17$ anys & 25,5 & 74,2 & 0,3 \\
$18-21$ anys & 31,8 & 67,7 & 0,5 \\
$22-25$ anys & 26,3 & 73,0 & 0,7 \\
$26-29$ anys & 27,0 & 73,0 & \\
\hline Activitat & & & \\
\hline Estudio & 28,1 & 71,7 & 0,2 \\
Estudio i treballo & 35,0 & 64,5 & 0,6 \\
Treballo & 25,9 & 73,9 & 0,2 \\
Atur & 14,3 & 84,5 & 1,2 \\
Feines de casa & 11,8 & 86,3 & 2,0 \\
Nivell d'estudis & & & \\
EGB incompleta & 21,0 & 78,2 & 0,8 \\
Graduat escolar & 19,3 & 80,7 & \\
FP & 21,8 & 78,2 & \\
BUP/COU & 31,0 & 68,8 & 0,2 \\
Estudis universitaris & 34,6 & 64,5 & 0,9 \\
Altres & 15,4 & 84,6 & \\
\hline
\end{tabular}

Fuente: Enquesta a la Joventut de Catalunya 1990.

A la pregunta de "En general, creus que per a un jove és interessant pertànyer a algun tipus d'associació?", un $29 \%$ de los jóvenes encuestados optaron por responder negativamente, frente a un $60 \%$ que contesta positivamente y un $11 \%$ que no sabe/no contesta. Pero la distancia entre esta actitud, en principio positiva, y una participación efectiva es muy notoria: del $60 \%$ de jóvenes inicialmente entrevistados que consideraban interesante en general pertenecer a una asociación, sólo un $28 \%$ manifiesta estar asociado. ¿Por qué más de un $50 \%$ de los jóvenes catalanes no traducen su actitud positiva hacia el asociacionismo en un comportamiento coherente con la misma?

Antes de abordar esta pregunta abierta creemos es necesario dejar constancia de los problemas que presentan las encuestas relativas a actitudes y comportamientos: la inevitable introducción de la subjetividad del propio 
entrevistado resta fiabilidad a las respuestas. En segundo lugar, a la pregunta sobre interés por el asociacionismo, se registró un $11 \%$ de ns/nc: las encuestas de opinión se basan en el error de imponer problemáticas sobre cuestiones que la mayoría de la población no se plantea y sobre las cuales, lógicamente, no tiene opinión. En el estudio que estamos utilizando, aparentemente, uno de cada diez jóvenes encuestados no tenía opinión sobre el hecho asociativo, pero sólo podemos suponer que los restantes sí tenían una opinión formada.

Para paliar estos problemas, Cardús y Estruch (1984) proponen abandonar deliberadamente el "culto a las estadísticas", utilizarlas como un medio secundario pero nunca como un fin en sí mismas: realizar un esfuerzo por vincular estos datos con la comprensión del porqué de los problemas, analizando no sólo sus manifestaciones sino también sus causas y consecuencias. Una vez relativizada la importancia de los datos cuantitativos, creemos que la pregunta que nos planteábamos - la distancia entre actitud y comportamiento asociativodeberíamos formularla de diferente manera: ¿Por qué se asocia ese $28 \%$ de jóvenes?

Lo que Villasante califica como «la penúltima moda del individualismo metodológico y de la elección racional» (1993: 9) reduce la complejidad de lo comunitario y asociativo a unos esquemas de orientación utilitarista. En esta corriente, Ronsenstone y Hansen (1993) plantean la participación como un análisis de costes y beneficios: la participación política tiene un precio para aquél que la practica, expresado como una combinación de dinero, tiempo, conocimientos y competencia política subjetiva. Sólo aquel que pueda "pagar este precio" estará en disposición de participar. Asimismo, quienes participan lo hacen pensando que obtendrán «algo a cambio». Las recompensas adquieren diferentes formas: beneficios materiales y/o intangibles (status, amistad, satisfacción personal).

La participación política entendida como elección racional está bien para el homo economicus. La naturaleza humana es mucho más rica, solidaria y creativa, así lo demuestran, por ejemplo, las acampadas populares por el $0,7 \%$ y la respuesta internacional contra la reanudación de las pruebas nucleares francesas en el Pacífico. Ambos movimientos han estado soportados por gran número y variedad de asociaciones -ecologistas, sindicales, de solidaridad, pacifistas, movimientos cristianos, asociaciones de vecinos., ONG, etc. - y plataformas unitarias, en un verdadero pulso a las instituciones.

¿Cómo explicarlo desde la teoría de la elección racional? Según sus teóricos, este tipo de acción política, en la cual los participantes reciben sólo beneficios colectivos y difusos de su actuación, si ocurre, sería irracional (Rosenstone y Hansen, 1993). Y sin embargo, las movilizaciones están en la calle y los afiliados siguen colaborando voluntariamente, en nombre de la ecología, la justicia social y la paz.

El error de fondo de este enfoque es que su sujeto de análisis es el hombre como ser racional, obviando la consciencia, las necesidades de expresión y los sentimientos de las personas, y el hombre tomado como individuo aislado, sin tener en cuenta su entomo ni su red de relaciones sociales. Almond y Verba 
(1963) demuestran cómo las actitudes y los comportamientos políticos están estrechamente relacionados con valores sociales y psicológicos. Estos autores consideran el sentimiento de competencia subjetiva y los valores sociales de cooperación y confianza interpersonales como los dos requisitos básicos para la explicación de la formación de grupos primarios y asociaciones voluntarias. Creemos de importancia desarrollar estos dos puntos para comprender y analizar nuestro objeto de estudio.

Sentimiento de competencia subjetiva. Las participaciones no políticas en la vida cotidiana (en la familia, la escuela, el trabajo, etc.) pueden dotar al individuo de la experiencia y confianza necesarias que le predispongan a adoptar un rol político activo. Almond y Verba (1963) observan un "orden jerárquico" en la fuerza de la relación entre diversas maneras de participación política: las experiencias profesionales en el trabajo y en las asociaciones voluntarias se distinguen de las ocurridas en la familia y la escuela por estar más vinculadas al sistema político. En primer lugar porque son contemporáneas con la participación política, el individuo ya ha acumulado cierto grado de experiencia y de madurez fuera del hogar; y en segundo lugar, porque en su forma se acerca más a los modos formales de participación política.

Pero cuando la apatía y la baja participación son los rasgos principales de la cultura política española, y dejando al margen la negativa experiencia de cuarenta años de dictadura, :podría ser que la familia y la escuela, como instituciones de socialización temprana, representasen un entrenamiento inadecuado para la participación política?

No podemos profundizar en este tema, sólo quisiéramos llamar la atención sobre la importancia del mismo con un par de observaciones. El núcleo familiar es el principal responsable de la reproducción de comportamientos patriarcales y paternalistas que siguen minando nuestro sistema social, introduciendo actitudes jerárquicas, autoritarias y discriminatorias que alcanzan todos los ámbitos de la vida social. Por lo que respecta al sistema educativo, se ha dejado a un lado la formación en el conocimiento como objetivo para priorizar la centralidad del trabajo como meta individual, no colectiva, del estudiante (titulaciones, másters, etc.). Desde la universidad podemos intuir cómo se ha producido un cambio en el valor del conocimiento per se, la formación humanística se ha trivializado, la educación se está reduciendo no a la mejora de las capacidades, sino al aumento de la competitividad del individiuo para encontrar un puesto de trabajo. El mensaje de la competitividad está calando socialmente, precisamente en el colectivo más perjudicado por esta trampa social: los jóvenes, los estudiantes de hoy.

Por lo que respecta a la socialización en el ámbito laboral, aunque importante y necesaria, nuestra hipótesis es que el actual mercado laboral no es el más apropiado para que el trabajador aporte iniciativas ni desarrolle su competencia subjetiva, muy especialmente en lo que respecta a jóvenes y mujeres: colectivos especialmente afectados por el paro y la precariedad laboral. Recordemos que uno de cada tres trabajadores en España está contratado temporalmente $(34,72 \%$ población ocupada) y que de cada cuatro jóvenes con- 
tratados, tres lo son de forma temporal $(63,8 \%)^{4}$. Para Cataluña, las cifras no varían sustancialmente.

Esta reflexión podemos ilustrarla en la escasa afiliación sindical juvenil: ser joven, especialmente menor de 25 años, es una característica que camina en paralelo con una baja tasa de afiliación (5\%), siendo principalmente atribuible a las características del mercado de trabajo. La misma afirmación es válida para el colectivo femenino $(27,4 \% \text { afiliadas })^{5}$, pero es necesaria una matización: el hecho de que tres de cada cuatro afiliados sean hombres no debe extrañarnos si tenemos en cuenta la baja tasa de actividad femenina española, diez puntos inferior a la media europea. A nivel estatal, las activas suponen algo más de un tercio de las mujeres en edad laboral (36,08\%), siendo este porcentaje un poco superior en Cataluña ( $40,76 \%)$, mientras que los varones presentan una tasa de actividad superior a dos tercios. Apuntamos la idea de que la experiencia laboral más que reforzar las actitudes participativas las debilita, al mismo tiempo que contribuye a conformar identidades «infra» en aquellos colectivos que ocupan las posiciones más bajas del status ocupacional: los jóvenes y las mujeres.

$\mathrm{Al}$ margen de los canales de socialización del individuo, una variable, el nivel educativo, es considerada como el determinante más importante de las actitudes políticas, pero no sabemos hasta qué punto podría suplir una socialización política deficitaria previa. En la tabla 1 podemos observar cómo el porcentaje de asociados aumenta en los niveles superiores educativos, independientemente de otras variables demográficas. Hay toda una serie de razones que explican esta relación, entre otras que el individuo educado también es más seguro de sí mismo y de su propia capacidad, por lo que debemos suponer que su competencia política subjetiva también es mayor. Pero no hay que olvidar que las diferencias de educación suelen estar vinculadas a otras características sociales: mayores posibilidades de poseer un status de ocupación más alto, de poseer ingresos superiores, también podría traducirse en más tiempo libre (no hay necesidad de hacer horas extras ni de pluriemplearse), etc.

Cooperación y confianza interpersonal. Pero la predisposición a asociarse no se basa solamente en las actitudes políticas del individuo, sino también en las actitudes con respecto a sus conciudadanos: es necesario un sentimiento de colectividad, de confianza interpersoral, un conjunto de valores de convivencia que predispongan a la colaboración por encima de los fines. El sentido de comunidad política es complejo de describir e imposible de aprender mediante los mismos canales de socialización que moldean nuestras actitudes políticas. Pero si el sentido de comunidad implica un sentimiento de identidad entre

4. Datos referidos al segundo trimestre de 1995. Fuente: INE, Encuesta de Población Activa.

5. Los datos sobre afiliación han sido extraidos del estudio no 5 del CERES, sobre la afiliación de las CC.OO. de Cataluña (CONC). Son datos de uno de los sindicatos mayoritarios, pero debemos suponer que los porcentajes serán muy similares a los datos sobre afiliación del resto de centrales. 
Tabla 2. Preferencias de los jóvenes a la hora de asociarse, en tanto por ciento y por tipos de asociación

\begin{tabular}{llll}
\hline & $\begin{array}{l}\text { Considera } \\
\text { molt interessant } \\
\text { pertànyer a } \\
\text { una associació }\end{array}$ & $\begin{array}{l}\text { Li agradaria } \\
\text { pertànyer a } \\
\text { una associació }\end{array}$ & $\begin{array}{l}\text { Pertany } \\
\text { a una } \\
\text { associació }\end{array}$ \\
\hline Tipus d'associació & 34,8 & 17,8 & 20,7 \\
Cultural & 34,7 & 22,6 & 30,4 \\
Esportiva & 5,6 & 2,2 & 7,8 \\
Religiosa & 40,1 & 19,9 & 11,7 \\
Excursionista & & 1,3 & 2,6 \\
De veïns & 4,9 & 3,2 & 5,4 \\
Política & 24,6 & 5,9 & 5,4 \\
De serveis cívics & & 0,3 & 1,1 \\
Sindical & & 15,6 & 3,4 \\
Ecologista & & 3,1 & 4,5 \\
De lleure & & 3,2 & 6,5 \\
Altres & &
\end{tabular}

Nota: el porcentaje de las dos primeras columnas está calculado sobre el total de la muestra; el de la tercera, sobre el total de jóvenes que pertenecen a una asociación. Fuente: Enquesta a la Joventui de Catalunya 1990.

conciudadanos, parece muy dudoso que pueda desarrollarse en sociedades fragmentadas, política o socialmente. En este punto nos gustaría preguntamos si discursos como el de la competitividad no están rompiendo la identidad comunitaria, sobre todo en el segmento de jóvenes. Si los altos porcentajes de paro y la precariedad laboral, concentrada en el colectivo femenino y juvenil, no estará minando también la identidad social de estos colectivos.

En relación con esto último, atendiendo a la variable ocupación en la tabla 1 observamos cómo el colectivo de los desempleados y de quienes se dedican a las tareas del hogar registran los menores porcentajes de afiliación (14,3\% y $11,8 \%$, respectivamente). Por sexos, las mujeres muestran tasas de asociación alrededor de 10 puntos inferiores a los hombres.

Pero, jen qué tipo de asociaciones se afilian los jóvenes catalanes? Son las entidades culturales y de ocio las que capitalizan su atención: aproximadamente, dos de cada tres jóvenes asociados $(62,8 \%)$. Este comportamiento podría decirse es coherente con el interés y la actitud favorable que muestran los encuestados a participar en asociaciones de este tipo.

Se trata de clubs deportivos, excursionistas, clubs de montaña, fundaciones, centros de estudios, ateneos, casa regionales... asociaciones con objetivos culturales, de animación, ocio y deporte, pero que podríamos resumir como "asociaciones de barrio". En efecto, se trata en su mayoría de asociaciones creadas en el ámbito local, ya que su principal atractivo está en su cercanía — no te 
desplazarás muy lejos para jugar un partido de fútbol o pasar un rato en tu casa regional - y en la inmediatez de sus fines - emplear el ocio en actividades que permiten estar en compañía cle un círculo de personas que pertenecen a tu entomo social, ya sean amigos, vecinos, familiares o compañeros de trabajo- sin más responsabilidades.

El asociacionismo cultural y recreativo se corresponde con un rasgo muy característico de nuestra cultura: el "gregarismo". Aunque algunos especialistas han afirmado que la cultura española se caracteriza por un alto grado de individualismo y escaso sentido del deber cívico, al mismo tiempo, se nos considera un pueblo gregario y festivo, que da mucho valor a las relaciones amistosas (Gunther, 1992).

De lo que no hay duda es de que, a pesar de su carácter no-político, la pertenencia a estas asociaciones representa un entrenamiento potencial para el compromiso y la actividad políticas: en la medida en que el individuo está expuesto, voluntariamente, a una riqueza de interacción social es de esperar que la afiliación tenga un efecto positivo sobre su competencia política (Almond y Verba, 1963).

Los porcentajes de afiliación del resto de opciones se sitúan muy por debajo, siendo aquellas alternativas que tienen un carácter más político o reivindicativo - asociaciones de vecinos, políticas y sindicales - las que peores resultados obtienen. Debido a que los jóvenes manifiestan poco interés por la política, pocos reflexionan sobre la importancia de su influencia política como ciudadano y, lógicamente, pocos también son los que se asocian en este tipo de organizaciones.

Por lo que se refiere a la asociaciones clasificadas por la Enquesta a la Joventut de Catalunya como de "Serveis cívics" entendemos que, de acuerdo con la clasificación de Alberich (1993), esta etiqueta comprende asociaciones de voluntariado y asistenciales —Cruz Roja, Cáritas, ayuda a minusválidos físicos y psiquicos, a drogodependientes, prevención de toxicomanías, asociaciones antisida, etc.- , pero no estrictamente de solidaridad ni pro-derechos humanos, las cuales no aparecen reflejadas en la tabla. "Serveis cívics" es receptora de cierto interés y actitud favorable por parte de los encuestados, creemos que por la buena prensa que se le está dando a las labores de voluntariado por parte de las instituciones y los medios de comunicación. Sin embargo, los índices de afiliación no se corresponden con el interés despertado, posiblemente porque la dedicación a estos objetivos asistenciales requiere un nivel de compromiso y responsabilidad hacia los demás que muchos no están dispuestos a asumir, a pesar del status que lleva consigo la labor.

Sean unas u otras las motivaciones, lo que nos dice el sentido común es que la gente se asocia para compartir: para encontrar en los otros lo que no encuentra en soledad -compañía, conversación, festejos...- y para satisfacer su necesidad de pertenencia, enriquecerse con otras experiencias y forjarse una identidad en comunidad. Los más conscientes se asocian, además, para luchar. 


\section{El "problema del trabajo»: ¿desarticulador social?}

Apuntábamos la hipótesis de que las experiencias laborales en el marco de trabajo actual debilitan las actitudes participativas, al mismo tiempo que contribuyen a conformar identidades "infra» en aquellos colectivos que ocupan las posiciones más bajas del status ocupacional: los jóvenes y las mujeres. En este apartado intentaremos desarrollar estas ideas, pero primero vamos a situarnos correctamente en el espacio y el tiempo.

La salida de la crisis de los setenta supondrá un enorme cambio en las estructuras sociales occidentales que, en gran medida, podría resumirse en el fenómeno de la "fragmentación" (Miguélez, 1990). El capitalismo de los ochenta abandona las políticas keynesianas en favor de las de oferta, legitimado por un discurso neoliberal, conformándose una nueva sociedad «dual, centrífuga y segmentada" (Alonso, 1992), donde el mercado se constituye como el gran regulador de todos los ámbitos de la realidad social.

En este contexto algunos autores han identificado con relación al desempleo un fenómeno de "transición del viejo al nuevo paro", caracterizado por un cambio en sus causas, consecuencias y en los propios protagonistas. Algunos sociólogos, ante las altas tasas de desempleo y los desequilibrios interregionales que, en general, se están manifestando en las economías occidentales, hablan de una nueva "sociedad dual" en términos empleados/desempleados. No obstante, esta supuesta dualidad debería matizarse atendiendo a un complejo mercado de trabajo donde conviven diferentes tipos y niveles cualitativos de empleo, ocupando la categoría más baja aquellos que J.M. Blanch (1990) ha calificado como los «infraempleados" en condiciones precarias, mal remuneradas, poco reconocidas, etc.

[...] los nuevos parias son los desempleados muy difícilmente empleables, concentrados en bolsas sociales de pobreza económica y marginación cultural, permanentemente nutridos por legiones crecientes de jóvenes fracasados escolares, encronizados en una transición hacia ninguna parte y excluidos, a menudo, de los programas de fomento del empleo - cuando éstos son aplicados según criterios de éxito previsible a corto plazo (J.M. Blanch, 1990: 277).

El discurso de la flexibilidad: aumento de la precariedad y segmentación de los trabajadores. Un nuevo modelo de producción altamente tecnológica y especializada, el postfordismo, intentará introducir la suficiente flexibilidad en la organización del trabajo para satisfacer las nuevas demandas sin perder los niveles de productividad fordista. El discurso neoconservador hará de la flexibilidad, y en especial de la flexibilidad laboral, su bandera, tras la que se camufla una discusión que afecta a la distribución de la renta y una estrategia orientada a liquidar una serie de mecanismos institucionales favorables a la clase obrera: la regulación de la acción sindical, las formas de contratación laboral y el recorte de los subsidios sociales (Recio, 1988). En España, las demandas empresariales de flexibilidad se instrumentalizaron en el R.D.18/1993 y afectaron especialmente al colectivo juvenil. 
La lucha de los jóvenes por insertarse en un mercado laboral cada vez más estrecho, inseguro y competitivo es a menudo infructuosa: a nivel estatal, aproximadamente uno de cada dos parados es menor de 25 años $(41,78 \%)$. En Cataluña esta tasa de paro es algo inferior $(38,57 \%)^{6}$. La última reforma del mercado laboral, con origen en el Real Decreto-Ley 18/1993, convertido después en la Ley 10/1994, pretexta facilitar la incorporación laboral de los jóvenes. Los objetivos a perseguir fueron facilitar, abaratándolos, los mecanismos de entrada y salida - para todo el colectivo trabajador - del mercado laboral, básicamente instrumentalizando dos nuevas modalidades de contratación: el contrato de aprendizaje y el contrato en prácticas.

Esta mal denominada reforma consistió esencialmente en la modificación de la Ley del Estatuto de los Trabajadores de 1980, violando el principio de suficiencia (art. 35.1.) y el principio de igualdad (art. 14) constitucional. No vamos a detenernos en comparar la regulación anterior al R.D.18/1993 con los cambios introducidos por la reforma ${ }^{7}$, pero sí nos interesa analizar sus consecuencias. Al margen de si aumentaron o no las contrataciones, en palabras de Franciso Pérez Amorós "[...] de mantenerse en los términos establecidos - la reforma - ser originaría una nueva fractura entre los trabajadores, puesto que se consolidaría un colectivo que además de ser asalariados temporales, resultarían discriminados tanto en los salarios como en la protección social. Además de ser desintegradora en sí misma, esta situación comportaría transmitir a los jóvenes una cultura del trabajo basada en la precariedad " ${ }^{8}$. Recordemos que uno de cada tres trabajadores en España está contratado temporalmente $(34,72 \%)$. Este nivel de temporalidad duplica el existente en Grecia y Portugal, y triplica la media del resto de países comunitarios. De cada cien jóvenes menores de 30 años contratados, el 63,8\% lo es temporalmente.

Esta fragmentación de la clase trabajadora y la consiguiente dualización social que comporta -algunos especialistas hablan de los nuevos parias- está ampliando el número de colectivos desorganizados y desprotegidos sindicalmente, porque su propia falta de cohesión dificulta en gran medida su organización y su representación: trabajo inmigrante, a domicilio, subcontratado, temporal, etc. La desarticulación de estos nuevos marginados es complementada con el efecto paralizante de la amenaza del paro: estos trabajadores, que dan pocos problemas, son perfectos para cubrir empleos precarios, mal retribuidos $\mathrm{y}$ con pocas perspectivas.

Desempleo. El "nuevo paro" tiene un fuerte componente juvenil y femenino. En el primer trimestre de 1995, la EPA registraba una tasa de paro del 23,52\% sobre la población activa: 3.647 .600 personas. El paro juvenil se sitúa en el $32,3 \%$, representando los activos menores de 25 años un $17,4 \%$ del total de

6. Datos referidos al segundo trimestre de 1995. Fuente: INE, Encuesta de Población Activa.

7. Para mayor información véase el cuadro comparativo elaborado por el Gabinete Jurídico Confederal de CC.OO., en L'Ona Sindical no 20, diciembre de 1993.

8. Cuatrosemanas y Le Monde Diplomatique no 14, marzo de 1994. 
población activa. Pero quizá el dato más angustioso son el medio millón de jóvenes menores de 25 años que son parados de larga duración y que de éstos, 300.000 llevan en paro más de dos años. Por último, la situación se agrava entre aquellos que buscan su primer empleo, pues el $74 \%$ de ellos se incluyen en esta misma categoría de desempleados de larga duración.

El grupo entre 25 y 29 años no engrosa estrictamente las cifras de paro juvenil, pero también merece nuestra atención porque aún podemos calificar a este colectivo como joven. Según algunos especialistas, estos jóvenes habrian visto empeorar relativamente su situación respecto a los otros grupos de menor edad, debido en parte a una sobreatención de las políticas de empleo hacia estos últimos y al efecto discriminatorio en función de la edad que ejercen algunos modelos contractuales - los actuales de aprendizaje y de prácticas, por ejemplo, de cuyos efectos ya hemos hablado-. Sea como fuere, los jóvenes están viendo como se "eternizan" en su condición de jóvenes dependientes económicamente del núcleo familiar, con todos los conflictos de identidad que esta situación conlleva. Algunos autores hablan de la tendencia a una «juvenilización de la pobreza» (Santos Ortega y otros, 1993).

Nuestra tasa de paro está entre las más altas de la Comunidad, aunque el aumento de la pobreza, el desempleo y, en particular, el desempleo de larga duración, es un fenómeno generalizado en los actuales estados de bienestar europeos. El aumento de esta presión sobre los sistemas tradicionales de indemnización por desempleo, ha forzado un replanteamiento: a la lógica de la prestacion económica se está incorporando una lógica de inserción del parado, acompañada de políticas de rentas mínimas. Buena parte de estas políticas están dirigidas a jóvenes, pero siguen basándose en valores capitalistas, individualismo y competencia, formándoles para competir mejor, no siendo ésta una solución real al problema de la exclusión ni de la explotación de estos colectivos (Santos Ortega y otros, 1993).

Los efectos sociales del desempleo, particularmente la exclusión social, no se derivan únicamente de una insuficiencia de ingresos. El trabajo remunerado, como institución sociocultural, no es sólo fuente de renta sino que también lo es de una serie de experiencias que, de ser negadas, producen una serie de carencias psicológicas en el desempleado: la pérdida de la estructura temporal culturalmente impuesta y de una actividad regular, la reducción de los contactos sociales, una pérdida de status y de identidad, la falta de participación en objetivos y esfuerzos colectivos. Querríamos destacar dos de estos fenómenos por su importancia: el deterioro del status/identidad y la exclusión de la participación en objetivos y metas colectivas.

El entorno laboral es fuente de roles, status e identidad para el trabajador. La "cultura del trabajo" (J.M. Blanch, 1990), tan propia de nuestras sociedades modernas, ha desplazado el punto de gravedad hacia el "hacedor", perdiendo interés por la persona en sí misma. Pero mientras que el status es un fenómeno esencialmente social, la "etiqueta" que nos atribuye el sistema de valores sociales, la identidad es un concepto más personal, íntimo y subjetivo. Sin embargo, ambas percepciones son interiorizadas de igual modo por el indivi- 
duo, con lo que los trastornos aparecen cuando al desempleado se le retira su status de trabajador.

La pregunta ahora es si una persona que duda de su propia identidad - una identidad que, no olvidemos, tiende a girar en torno del trabajo como razón de la existencia- puede identificarse con esos «otros». El empleo, en teoría, es un contexto de afiliación, participación, interacción grupal, comunicación... pero este entrenamiento en las actitudes participativas y asociativas ¿se pierde porque el desempleado no encuentra "su» colectivo ni ningún objetivo ni meta colectiva que perseguir? El grado de desequilibrio que pueda significar para cada desempleado estás carencias, variará en función de si el individuo dispone de otras compensaciones en su entorno: actividades alternativas donde ocupar su tiempo, apoyo social, clima familiar, etc. Depende de ellos mismos hallar vías alternativas que le aporten la estabilidad psicológica y social que ahora le faltan, en su propia vida privada o en otras organizaciones.

M. Jahoda (1987) Ilega a la conclusión de que las necesidades psicológicas que el empleo satisface son probablemente más profundas y permanentes que los mecanismos institucionales que las satisfacen. Sin embargo, en ocasiones la calidad negativa de las experiencias que se tienen en el empleo puede ser de tal magnitud que el desempleo sería preferible para la estabilidad psicológica de estas personas: todos los especialistas están de acuerdo en que los trabajadores manuales están sometidos a terribles presiones temporales - trabajo a destajo, procesos de producción en cadena - y sufren continuas humillaciones para hacerles sentir cuál es su puesto en la jerarquía del sistema. Los informes de estudiosos del tema como Palm (1977), Linhart (1976) y Pfeffer (1979) coinciden en reconocer el desprecio y la desconfianza hacia el trabajador manual como intrínsecos al sistema, a lo que éstos responden con un fuerte resentimiento y un reforzamiento de su identidad de clase.

Si a estas presiones y vejaciones sufridas por los trabajadores manuales en las industrias fordistas, añadimos las propias de la etapa actual: inseguridad en el trabajo; fragmentación de la clase trabajadora y pérdida de identidad de colectivos en situaciones intermitentes de empleo/desempleo/subempleo; relajación de la solidaridad (por ejemplo: temática de las horas extras) entre compañeros de trabajo que ahora también son vistos como "competidores»; desorganización y desprotección legal y sindical de algunos colectivos difíciles de cohesionar (inmigrantes, subcontratados, etc.); etc., la pregunta es ipuede hablarse de participación en objetivos y metas colectivas en los centros de trabajo? En las circunstancias actuales ¿son las experiencias derivadas de relaciones laborales un buen entrenamiento para fomentar las actitudes participativas $y$ asociativas? ¿Aumentan o disminuyen la confianza interpersonal?

Pero si estos son los desequilibrios que la cultura del trabajo en precario está transmitiendo, en especial, a los jóvenes, igual de preocupantes son los sentimientos de violencia y frustración que experimentan los jóvenes parados de larga duración. 
La desesperación y el pesimismo iniciales se transformaban, con el transcurso de ese período de tiempo, en resignación y apatía, lo que recuerda al proceso que tan a menudo se manifestó en los años treinta. Pero existe una diferencia. La resignación y la apatía de estos jóvenes quedaban indicadas en la falta de esperanza en el futuro y en el abandono de la búsqueda de un puesto de trabajo después de repetidos fracasos; pero aparentemente no se daba ninguna reducción en una hostilidad que, a falta de un blanco al que claramente pudieran culpar de su situación, se dirigía difusamente contra toda la comunidad (Stokes, 1981, citado en M. Jahoda, 1987: 79).

Podría pensarse que aquellos que gozan de un mejor nivel educativo se hallarán en mejor situación de superar estos transtomos psicosociales. Sin embargo, es el colectivo mejor educado el que tiene más posibilidades de haber desarrollado unas expectativas excesivas, luego no correspondidas en el mercado laboral. El subempleo o desempleo en estas situaciones puede ser más difícil de sobrellevar psicológicamente, a pesar de que la persona educada haya tenido más oportunidades de desarrollar otras identidades y recursos que le compensen de esta mayor frustración. No obstante, sabiendo de la centralidad que ocupa el «Trabajo» en la formación académica del individuo, hallar alternativas e impedir que esta frustración derive en sentimientos como hostilidad, rencor y resentimiento hacia el sistema, creemos es mucho pedir.

\section{Algunas conclusiones}

Si el pluralismo es uno de los fundamentos más importantes de la democracia, las asociaciones juegan un papel de primer orden en el sistema democrático como puente de contacto entre la sociedad civil y el Estado. Las asociaciones voluntarias se conforman como «microdemocracias» que beben directamente del pulso popular, evolucionando en sus identidades al tiempo que evolucionan los movimientos a los que soportan y sobre los que construyen sus valores. Son a la vez conformadoras y garantes del consenso democrático, e introductoras del cambio social: con esta doble función contribuyen al dinamismo de la propia democracia.

Los estudiosos de la cultura política de los españoles coinciden en señalar nuestro carácter escasamente participativo e individualista. Los bajos niveles de asociacionismo juvenil son coherentes con este talante, y no puede calificarse como un rasgo generacional. Esta podría ser una explicación estructural, pero nuestra hipótesis es que, muy estrechamente relacionado con la fragmentación social y la precarización laboral —de colectivos juveniles y femeninos, pero también inmigrantes y otros "grupos de riesgo»-, se está desarrollando una "cultura de la precariedad» que amenaza las bases del asociacionismo y de la propia participación política.

Las participaciones no políticas en la vida cotidiana pueden dotar al individuo de la experiencia y la confianza necesarias que le predisponga a adoptar un rol político activo. De especial importancia son las experiencias profesio- 
nales en el trabajo y en las asociaciones voluntarias, ya que éstas son contemporáneas y se realizan por canales más formales, características que las vinculan en tiempo y modo de participación al sistema político.

Sin embargo, y pese a su centralidad, el actual mercado laboral no es el más apropiado para que el trabajador aporte iniciativas ni desarrolle su competencia subjetiva, muy especialmente en lo que respecta a jóvenes y mujeres, colectivos éstos gravemente afectados por el desempleo y la precariedad laboral. Como resultado de la calidad negativa de sus experiencias, los «infraempleados» van a transmitir una cultura del trabajo basada en la precariedad que va a influir en las actitudes políticas negativamente, y que va a tener en el propio núcleo familiar un excelente canal de socialización (las mujeres, trabajadoras o desempleadas, siguen ejerciendo una poderosa influencia en el hogar). Esta "cultura de la precariedad" de los nuevos parias es diametralmente opuesta a las condiciones entendidas como necesarias para fomentar el asociacionismo y la participación política en sentido amplio: la competencia subjetiva y la confianza interpersonal.

Un trabajador cuando sabe que está siendo explotado y no tiene posibilidades de organizarse para defenderse o abandonar su trabajo, es plenamente consciente de que su lugar está «abajo», y frente a este sentimiento de impotencia sólo cabe la hostilidad y el rechazo hacia el sistema. El desarrollo de competencia política por parte de un sujeto frustrado y solo en su angustia es muy improbable.

Por otro lado, si el sentido de comunidad implica un sentimiento de identidad entre conciudadanos, es dudoso que pueda desarrollarse en sociedades fragmentadas y desarticuladas. Discursos como el de la competitividad y el individualismo están desgarrando el sentimiento de comunidad y las señas de identidad y pertenencia de los ciudadanos. La pregunta no es con quién nos identificamos, sino contra quién compito.

En esta nueva etapa capitalista de abandono del keynesianismo, que está retomando el protagonismo indiscutible al mercado sobre la sociedad civil, el nuevo discurso conservador culpa a los trabajadores y a sus representantes, e introduce nuevos mensajes y valores que van a erosionar las identidades de clase y los sentimientos de pertenencia comunitarios. El mensaje es la competitividad y la flexibilidad laboral; los valores, el individualismo y la eficiencia.

El sistema educativo, los medios de comunicación y las propias instituciones del Estado se han hecho eco de este discurso y han ensalzado la faceta del "hacedor" por encima de la persona como ser, esforzándose por etiquetarlo para controlarlo mejor, en base a sus actitudes y comportamientos, olvidando los sentimientos.

Los trastornos psicosociales que sufre el subempleado y el parado como consecuencia de su situacion laboral serían evitables si el «hacedor» creado por la opinión pública y formado en los centros de enseñanza, dejase paso a mujeres y hombres más libres, capaces de hallar otros mecanismos - las asociaciones voluntarias podrían ser la alternativa - para satisfacer sus necesidades psicológicas en su propia vida privada. Pero la lógica asociativa no entra den- 
tro de los esquemas del discurso neoconservador: frente al homo economicus y los análisis sociológicos que pretenden explicar los fenómenos asociativos en función de costes/beneficios, al margen valores sociales, las asociaciones son lugares donde la gente se reúne para compartir tiempo, experiencias y sentimientos.

Sin embargo, no todas las asociaciones voluntarias están al margen del discurso neoliberal. Las asociaciones consideradas de voluntariado y servicios cívicos están siendo mimadas por medios de comunicación e instituciones públicas. Dentro de una nueva estrategia que pretende recrear un escenario de la "sociedad de la actividad» (Santos Ortega, 1993) —ya que del pleno empleo y de la integración es imposible- en el que se contengan todas esas energías que el desempleo libera, se siga entronizando al trabajo en la jerarquía de valores y camufle el problema de manera que no se plantee un cambio de política radical. En sintonía con este discurso, se han definido una amplia gama de actividades de "inserción", alternativas a un empleo fijo y remunerado: actividades de formación, trabajos de "voluntariado", actividades de utilidad colectiva, la educación continuada, etc. son una constelación de actividades intermedias que maquillan la desocupación y mantienen el mensaje competitivo con todo su vigor.

\section{Bibliografía}

ALMOND, A.; VerBA, S. (1963). La Cultura Civica. Estudio sobre la Participación Politica Democrática en Cinco Paises. Euramérica.

ALONSO, L. "Postfordismo, fragmentación social y crisis de los nuevos movimientos sociales". Sociología del Trabajo, nueva época, núm. 16, otoño de 1992, p. 119-141.

Alberich Nistral, T. "Aspectos cuantitativos del asociacionismo en España". Documentación Social, núm. 90, enero de 1993. p. 53-74.

BARNES, S.; KAASE, M. (1979). «Political Action, a theoretical perspective» $y$ «Political action repertory», y INGLEHART, R. (1979). "Value priorities and socioeconomic change". Political Action, Sage, cap. 12, 5 y 11.

Blanch, J.M. (1990). Del Viejo al Nuevo Paro. Un Análisis Psicologico y Social. Barcelona: PPU.

Botella, J.; Cañeque, C.; Gonzalo, E. (1994). El Pensamiento Político en sus Textos. Madrid: Tecnos, p. 382-406.

CARDús, S.; EstruCh, J. (1984). Les Enquestes a la Joventut de Catalunya. Generalitat de Catalunya, p. 73-109.

Enquesta a la joventut de Catalunya 1990. Gabinet d'Estudis Socials. D.G.P., Generalitat de Catalunya, 1991.

FeIXA y PAMPOLS, C., (1993). La Joventut com a Metáfora. Generalitat de Catalunya, Secretaria General de la Joventut.

GunTher, R. (1992). Politica y Cultura en España. Madrid: Centro de Estudios Constitucionales.

JAHODA, M. (1987). Empleo y Desempleo: un Análisis Socio-Psicológico. Madrid: Ediciones Morata.

LIPSET (1987). El Hombre Politico. Madrid: Tecnos, p. 22-36.

Pennock, R. (1979). Democratic Political Theory. Princeton University Press, p. 206$259 ; 438-469$. 
Pérez Olivares, E. (1989). La Democracia Participativa. Quito: FESO (Fundación Ecuatoriana de Estudios Sociales).

Rebollo, O.; Martín, A.; Miguélez, F. (1993). El Sindicalismo a través de sus Protagonistas. Estudio sobre la Afiliación de CONC. Barcelona: CERES.

RECIO, A. «FIexibilidad, eficiencia y desigualdad (notas sobre la flexibilidad laboral)». Sociología del Trabajo, nueva época, núm. 4, otoño de 1988. p. 81-105.

RosENSTONE y HANSEN (1993). Mobilization. Participation and Democracy in America. Nueva York: Macmillan, cap. 2.

SANTOS ORTEGA, J.A.; RoDRíGUEZ, M. «La sociedad de la inserción: trabajo, paro y tratamiento social de la exclusión». Sociología del Trabajo, nueva época, núm. 18, primavera de 1993. p. 99-118.

VILLASANTE, T. «Los retos del asociacionismo». Documentación Social, núm. 90, enero de 1993, p. 9-21. 